\title{
An Analytical and Spatial Model of Foraging in a Swarm of Robots
}

\author{
Heiko Hamann and Heinz Wörn \\ Institute for Process Control and Robotics, \\ Universität Karlsruhe (TH) \\ 76128 Karlsruhe, Germany \\ \{hamann, woern\}@ira.uka.de
}

\begin{abstract}
The foraging scenario is important in robotics, because it has many different applications and demands several fundamental skills from a group of robots, such as collective exploration, shortest path finding, and efficient task allocation. Particularly for large groups of robots emergent behaviors are desired that are decentralized and based on local information only. But the design of such behaviors proved to be difficult because of the absence of a theoretical basis. In this paper, we present a macroscopic model based on partial differential equations for the foraging scenario with virtual pheromones as the medium for communication. From the model, the robot density, the food flow and a quantity describing qualitatively the stability of the behavior can be extracted. The mathematical model is validated in a simulation with a large number of robots. The predictions of the model correspond well to the simulation.
\end{abstract}

Key words: macroscopic model, foraging, swarm robotics, mathematical analysis

\section{Introduction}

The ongoing advances in electronics and robotics have made it possible to build small robots of sizes below $3 \times 3 \times 3 \mathrm{~cm}^{3}$ at low cost. See website [6] for example which is part of the European project I-SWARM [18] and also gives an overview over other existing platforms. This evolution made it feasible to implement large groups of 50 or more robots. While the hardware is available, the development of the control software is still a problem. To minimize the complexity of the entire system, the development targets simple rules and, in an allusion to nature, one hopes for emergent behavior of the robot group that leads to the solution of the predefined task. However, both the design of the general strategy and the configuration of many influential parameters are in general not supported by any guideline based on theoretical results. Therefore, the software is just implemented using simple heuristics based on experience and a trial-and-error process. To fill this gap, a scientific basis that describes the behavior of robot swarms would be desirable.

A first step could be the development of analytical models, that support us in understanding the results obtained by simulations and experiments in a 
better way. Additionally, such models help to save resources, because they are usually faster than simulations and they give some insights before a single robot is implemented. The use of such models for finding optimal parameters is limited but possible, if a mapping from the abstract level to reality exists [13].

Although research in this field has just begun, a lot of results have been published recently: A probabilistic, analytic, and macroscopic model based on rate equations has been introduced and applied to several different scenarios by Lerman, Martinoli, Matarić, and others and performs very well in predicting the ratios of robots being in a certain state at a certain time $[9,12,11]$. It is based on the assumption that the space is uniform, so that for example scenarios involving pheromones cannot be represented using this model. However, there exist many models that approximate spatial characteristics: For example the dimensionality of space is reduced by modeling the movement of agents as a graph or a line of decision points [5,4], space is discretized using cellular automata [3], or space is fully but only microscopically modeled using Monte Carlo simulations [21, 2]. An analytical microscopic model with respect to space based on Brownian motion has been presented by Schweitzer $[15,16]$. In some cases the derivation of a macroscopic model from the microscopic descriptions has been performed and presented.

The foraging scenario is an old problem in robotics and hence it has been investigated intensively. There are many variants of this scenario: A single robot or a group of robots has to find and collect or basically only transport (food) items to some random locations or to one defined place. A brief overview of the work in robotics on this scenario is given in [10]. Here we focus on the situation having one place where food can be found (we will refer to this as food) and another place where the food should be delivered (nest). Our approach will make use of pheromones as the tool for communication and will utilize a large number of robots. In [14] an overview of simulations connected to this special scenario is given and an approach is presented using two dynamic pheromones, i.e. their distributions change over time.

An analytical macroscopic model for a variant of foraging is presented in [10]. The given rate equations are based on the assumption of homogeneous space and no pheromones are used in the investigated scenario. In [17,15], a spatial model for trail formation by ants using two pheromones is given. The model is based on differential equations that describe the agents microscopically.

\section{Simulation}

The analytical model will be validated with results of a simulation. As the framework for our simulation we use the Breve simulator by J. Klein [8]. Here, we simulate a homogeneous swarm in continuous space combined with a discrete implementation of the pheromones. Since we want to simulate big numbers of robots over many runs, we depend on a rather simple model of the robot, that is computationally easy to handle. In our model, the robot has circumferential 
visibility, can measure distances to objects within the coverage of its sensors, and can distinguish between other robots, the nest, food, and the wall, that circumscribes the arena. It is also able to perceive a pheromone gradient in two mutually orthogonal directions and to drop a certain amount of pheromone. The robots' locomotion is assumed to be ideal, i.e. an acceleration towards an arbitrary direction is possible at all times (holonomic drive mechanism). The control of the robot is totally reactive and based on the principle of virtual physics (potential field techniques), i.e. other objects have a repelling effect on it depending on their distance and visibility $[19,7,1]$. This defines the avoidance behavior to be similar to the collision of two particles in our real physical world.

A robot is in one of two possible states: looking for food $\left(s_{f}\right)$ or returning home to the nest $\left(s_{n}\right)$. Initially, all robots are randomly positioned close to the nest with a random velocity heading to a random direction and all start in state $s_{f}$. If a robot in state $s_{f}$ perceives the food, it transitions to state $s_{n}$. Robots in state $s_{n}$ perform a transition to state $s_{f}$, if they perceive the nest. We are using two pheromones: one that should be established to increase in intensity towards the food $\left(p_{f}\right)$ and another one that increases towards the nest $\left(p_{n}\right)$. To avoid immense instabilities and to simplify the scenario, pheromone $p_{n}$ is chosen to be present and constant at all times. It is always guaranteed to have a smooth gradient leading to the nest at any position. However, at least in a grid world based on a concept of dying agents it has been shown, as mentioned above, that a stable behavior can be reached with two dynamic pheromones with the advantage of finding shortest paths around obstacles [14]. As a second consequence, the robots will only be able to deposit the pheromone $p_{f}$, which they will do in state $s_{n}$. The amount that is dropped by the robot at each simulation step is set to an initial value (drop size, see Table 1) at the state transition and decreases exponentially over time thereafter (drop decrease rate).

The pheromones are implemented by a grid of so-called patches that is laid over the whole arena. The patches are quadratic and we have chosen a size of $s=6 \mathrm{~cm}$ (for comparison: a patch fits into the area covered by the robot's sensors). The performance of the swarm is independent of this size as long as it is reasonably small and both the evaporation and the diffusion rate are adapted to it. But choosing the patch size is computationally critical because the evaporation and diffusion process of pheromone $p_{f}$ is executed at every time step, which has to handle every single patch (complexity is $O\left(\frac{1}{s^{2}}\right)$ ), another option could be to update the grid only every $m$ time steps for $m>1$ ). Every patch has an associated pheromone intensity $i$ that is updated per step by

$$
i_{t+1}=(1-e-d) i_{t}+\sum_{n \in \mathcal{N}} n(1-e) \frac{d}{4},
$$

where $e$ denotes the evaporation rate, $d$ the diffusion rate and $\mathcal{N}$ the set of intensities of the patches in the von Neumann neighborhood of range one except the current patch itself. If a robot deposits some pheromone $p_{f}$, the dropped amount will be added to the intensity associated with the patch where the robot 
is located at that moment. Pheromone $p_{n}$ is time-invariant as discussed above (see Section 4 for the definition).

To implement the gradient ascending, the two components of the gradient are computed from the intensities of the neighboring patches independent of the robot's orientation: $g_{x}=n_{x+}-n_{x-}$, where $n_{x+}$ denotes the intensity of the neighboring patch in positive x-direction and $n_{x-}$ in negative x-direction; $g_{y}$ is computed analogously. The overall acceleration vector of the robot is a weighted sum of $\frac{\left(g_{x}, g_{y}\right)^{T}}{\left|\left(g_{x}, g_{y}\right)^{T}\right|}$ (if $\left|\left(g_{x}, g_{y}\right)^{T}\right|=0$ the term is set to 0$)$ and another vector depending on sensed objects that implements the avoidance behavior.

Table 1. Simulation parameters.

\begin{tabular}{|l|c|}
\hline Parameter & Value \\
\hline arena size & $258 \mathrm{~cm} \times 258 \mathrm{~cm}$ \\
nest position & $(129 \mathrm{~cm}, 195 \mathrm{~cm})$ \\
food position & $(129 \mathrm{~cm}, 63 \mathrm{~cm})$ \\
patch size & $6 \mathrm{~cm}$ \\
agent diameter & $2 \mathrm{~cm}$ \\
proximity sensor range & $5 \mathrm{~cm}$ \\
\hline iteration step & $0.05 \mathrm{~s}$ \\
max. speed & $7 \mathrm{~cm} / \mathrm{s}$ \\
evaporation rate & $0.03921 / \mathrm{s}$ \\
diffusion rate & $0.15681 / \mathrm{s}$ \\
drop decrease rate & $0.0951 / \mathrm{s}$ \\
initial drop size & 0.5 \\
\hline
\end{tabular}

\section{Analytical Model}

In [16] macroscopic equations are presented, that are derived from microscopic equations to describe the behavior of so-called "heatbugs". The "heatbug" simulation bears resemblance to the scenario addressed here: Corresponding to the pheromone, it is also a spatial property - the heat, that influences the movements of the bugs and which is also manipulated by them. We use the equation of the agent density from [16] as a starting point:

$$
\begin{aligned}
\frac{\partial}{\partial t} S(\mathbf{r}, t) & =D \frac{\partial^{2} S(\mathbf{r}, t)}{\partial \mathbf{r}^{2}}-\alpha \frac{\partial}{\partial \mathbf{r}}\left[\frac{\partial P(\mathbf{r}, t)}{\partial \mathbf{r}} S(\mathbf{r}, t)\right] \\
& =D \nabla^{2} S(\mathbf{r}, t)-\alpha \nabla[\nabla P(\mathbf{r}, t) S(\mathbf{r}, t)],
\end{aligned}
$$

where $S(\mathbf{r}, t)$ denotes the density of robots at position $\mathbf{r}$ at time $t, P(\mathbf{r}, t)$ the intensity of the pheromone, $D$ the diffusion constant, $\alpha$ the greediness of following the gradient, and $\nabla$ the gradient. 
The first term describes a standard diffusion process according to Fick's Second Law that models the exploring robots as well as robots avoiding collisions. The underlying assumption to motivate the application of this equation to the scenario investigated here is: If the density of robots in the arena is inhomogeneous then the robots tend to move away from spots of higher density into areas with less density. Please note that our approach of using virtual physics in our simulation meets this assumption. Thus, the diffusion term is a suitable mathematical description of robots performing some kind of random walk.

Because the rate of diffusion usually depends on the local density, the choice of using a constant diffusion coefficient is a simplification justified by two considerations: First, in the steady state the regions of highest density are most relevant and these densities reside within a small interval. Second, typically the diffusion constant has to be measured in an experiment or simulation. Having to measure the diffusion as a function of the density means higher overhead and demands longer running times to reach a reasonable accuracy.

The second term describes for $\alpha>0$ a gradient ascent of the robots proportional to the pheromone gradient. Although applications of the pheromone scenario could exist in which such a behavior of the robots might be desired, we drop the proportionality to the pheromone intensity of the gradient ascent, because this corresponds to a more efficient control software of robots and results in better stability in both the simulation and the used numerical solver of the analytical model. This is achieved by normalizing the gradient of $P$ to one:

$$
\frac{\partial}{\partial t} S(\mathbf{r}, t)=D \nabla^{2} S(\mathbf{r}, t)-\alpha \nabla\left[\frac{\nabla P(\mathbf{r}, t)}{|\nabla P(\mathbf{r}, t)|} S(\mathbf{r}, t)\right] .
$$

In the case of $|\nabla P(\mathbf{r}, t)|=0$ the second term is set to 0 . However, this model does not support several states, several pheromones, or state transitions and an extention is necessary (note that in [15] another way of incorporating internal states and several potential fields is given). First, we introduce the densities $S_{f}$ and $S_{n}$ that describe the densities of robots in state $s_{f}$ and $s_{n}$ respectively. Accordingly, we introduce the pheromone intensities $P_{f}$ and $P_{n}$ that correspond to the pheromones introduced in the previous section. This leads to two partial differential equations (PDE) in the same form as equation 4:

$$
\begin{aligned}
& \frac{\partial}{\partial t} S_{f}(\mathbf{r}, t)=D_{f} \nabla^{2} S_{f}(\mathbf{r}, t)-\alpha_{f} \nabla\left[\frac{\nabla P_{f}(\mathbf{r}, t)}{\left|\nabla P_{f}(\mathbf{r}, t)\right|} S_{f}(\mathbf{r}, t)\right], \\
& \frac{\partial}{\partial t} S_{n}(\mathbf{r}, t)=D_{n} \nabla^{2} S_{n}(\mathbf{r}, t)-\alpha_{n} \nabla\left[\frac{\nabla P_{n}(\mathbf{r}, t)}{\left|\nabla P_{n}(\mathbf{r}, t)\right|} S_{n}(\mathbf{r}, t)\right] .
\end{aligned}
$$

Now we are investigating a system with multicomponent diffusion but we are still using Fick's law that does not model the coupling of the two diffusion coefficients and is only exact for two components. However, this is a common approximation in physics and is the more accurate the higher the concentration of the supporting medium (here: space) is, which is a suitable assumption here. Thus these two PDE are coupled only indirectly by the state transitions and the pheromones, which will be defined in the following. 
The nest and the food are modeled as areas with special boundary conditions that implement the state transitions. Say $\partial \Omega_{n}$ is the boundary of the arena around the nest. Then we define the boundary conditions at the nest as the following:

$$
\begin{gathered}
\forall \mathbf{r} \in \partial \Omega_{n}: \frac{\partial S_{f}}{\partial t}(\mathbf{r}, t)=D_{f} \nabla^{2} S_{f}(\mathbf{r}, t)-\alpha_{f} \nabla\left[\frac{\nabla P_{f}(\mathbf{r}, t)}{\left|\nabla P_{f}(\mathbf{r}, t)\right|} S_{f}(\mathbf{r}, t)\right] \\
+D_{n} \nabla^{2} S_{n}(\mathbf{r}-\epsilon \mathbf{n}, t)-\alpha_{n} \nabla\left[\frac{\nabla P_{n}(\mathbf{r}-\epsilon \mathbf{n}, t)}{\left|\nabla P_{n}(\mathbf{r}-\epsilon \mathbf{n}, t)\right|} S_{n}(\mathbf{r}-\epsilon \mathbf{n}, t)\right] \\
\forall \mathbf{r} \in \partial \Omega_{n}: S_{n}(\mathbf{r}, t)=0,
\end{gathered}
$$

where $\mathbf{n}$ denotes the exterior normal to the boundary (pointing towards the nest center). The intuitive interpretation of these equations is simple: The robots in state $s_{n}$, that are close to the nest, perform a transition to $s_{f}$, because they have finished their mission to find the nest. In a trivial grid discretization of these PDE, the boundary conditions are implemented by adding the amount of $S_{n}$ to $S_{f}$ and setting $S_{n}=0$ within the area of the nest after each iteration. The boundary conditions at the food are defined in an analog way. The boundaries of the arena are modeled as total isolation.

The pheromone $P_{n}$ leading to the nest is assumed to be constant over time and is just defined as it is implemented in the simulation:

$$
P_{n}(\mathbf{r})=c_{1}\left(\sqrt{d_{\max }}-\sqrt{d\left(\mathbf{r}, \mathbf{r}_{n}\right)}\right),
$$

where $d\left(\mathbf{r}, \mathbf{r}_{n}\right)$ is the distance to the center of the nest, $d_{\max }$ the maximal possible distance, and some constant $c_{1}$ that is used to adapt $P_{n}$ to the absolute intensities of $P_{f}$ (see Table 2). However, every function that provides a gradient pointing towards the nest at all positions could be used, since our model as well as the simulation are both independent of the absolute values.

Pheromone $P_{f}$ is modeled to depend on $S_{n}$ directly:

$$
P_{f}(\mathbf{r}, t)=S_{n}(\mathbf{r}, t) c_{2}^{c_{3} d\left(\mathbf{r}, \mathbf{r}_{f}\right)},
$$

where $d\left(\mathbf{r}, \mathbf{r}_{f}\right)$ is the distance to the center of the food, and some constants $c_{2}<1$ and $c_{3}$ depending on the pheromone dropping procedure. The underlying consideration is that the amount of pheromone dropped per step by a robot decreases exponentially with time. Thus the amount of pheromone that can be dropped by a robot at a certain spot is limited by the time a robot needs to travel from the food to this spot. Assuming a constant velocity this time is proportional to the distance to the food.

Note that no dependency on the history of $S_{n}$ is incorporated. Intuitively, one might argue, that this direct coupling corresponds to a high evaporation rate of the pheromone and thus could cause instabilities in the modeled overall behavior of the swarm, that would not emerge for appropriate parameter settings. However, for reasonable values of diffusion $D$, the history is intrinsically modeled. For the steady state this is quantitatively true and can be shown 
under the assumption that at each patch the pheromone diffusion net flux is zero. Since the robots depend on the normalized gradient only, a qualitatively correct representation of the gradient is sufficient. For visualization, imagine a large group of robots in state $s_{n}$ starting at the food and moving towards the nest following the gradient greedily and leaving behind only few other robots that moved to different directions due to diffusion. On the line between food and nest they would create a pheromone trail starting high at the food and decreasing exponentially towards the nest because of the dropping method. Since the density of the robots left behind would not exponentially increase towards the nest, this situation is represented qualitatively correct in our model. Similar considerations imply that equation 10 is a good approximation.

Table 2. Pheromone parameters.

\begin{tabular}{|c|c|}
\hline Parameter & Value \\
\hline$c_{1}$ & $1.4 \cdot 10^{-4}$ \\
$c_{2}$ & 0.998 \\
$c_{3}$ & 10 \\
\hline
\end{tabular}

\section{Results}

To simplify the following investigations, we restrict ourselves to a special case and set $\alpha_{f}=\alpha_{n}:=\alpha$ and $D_{f}=D_{n}:=1-\alpha=D$. This class of parameter settings is of special interest, because for example one would expect that the configuration with the maximal flow of food belongs to this class of symmetric diffusion settings, which is also supported by the results of our simulations. Now only a single variable that connects the model to the simulation is left - the diffusion $D$.

This diffusion parameter might be directly extracted from the robot control, if the control method enforces a certain amount of diffusion. However, the diffusion will typically depend on the local density also and here the diffusion is not explicitly implemented in our control software. Thus it has to be determined from the overall behavior in the simulation. This was done by measuring the amount of robots that follow the pheromone gradient approximately in comparison to those that move to any other direction at many different positions in the arena. The average diffusion was computed by weighting these values by their local density averaged over time.

The solution of the PDE provides us basically with the stationary density distributions of robots in states $s_{f}$ and $s_{n}$, if they exist. Fig. 1(a) shows a typical solution and Fig. 1(b) the corresponding averaged density of 30 simulation runs. The accuracy of the model is good at positions between nest and food. However, close to the nest and the food it suffers from the unmodeled acceleration processes of the robots and the impossibility to represent the infinite slope of the densities in the simulation. 


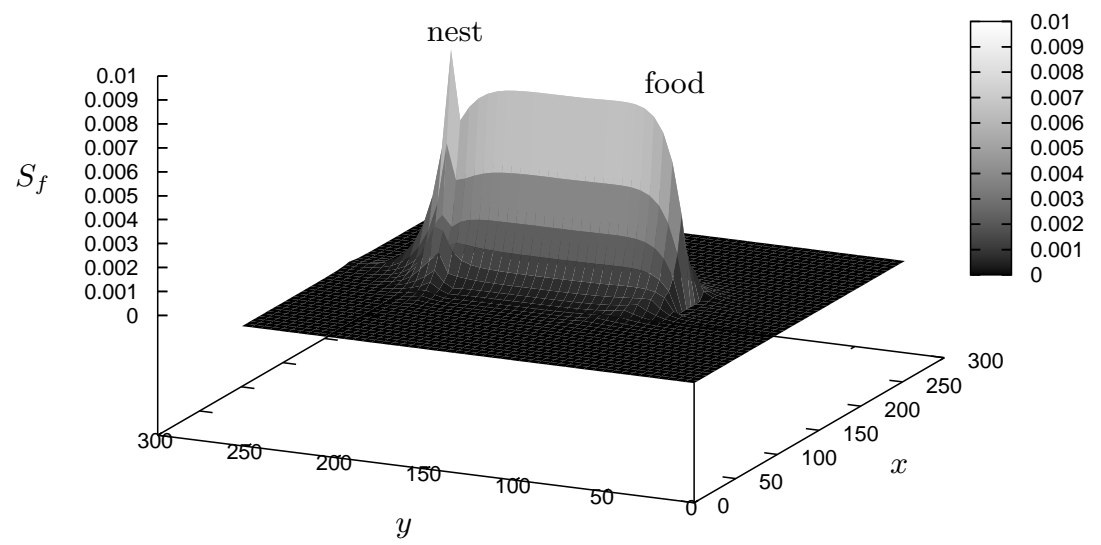

(a) Prediction of the model for the steady-state.

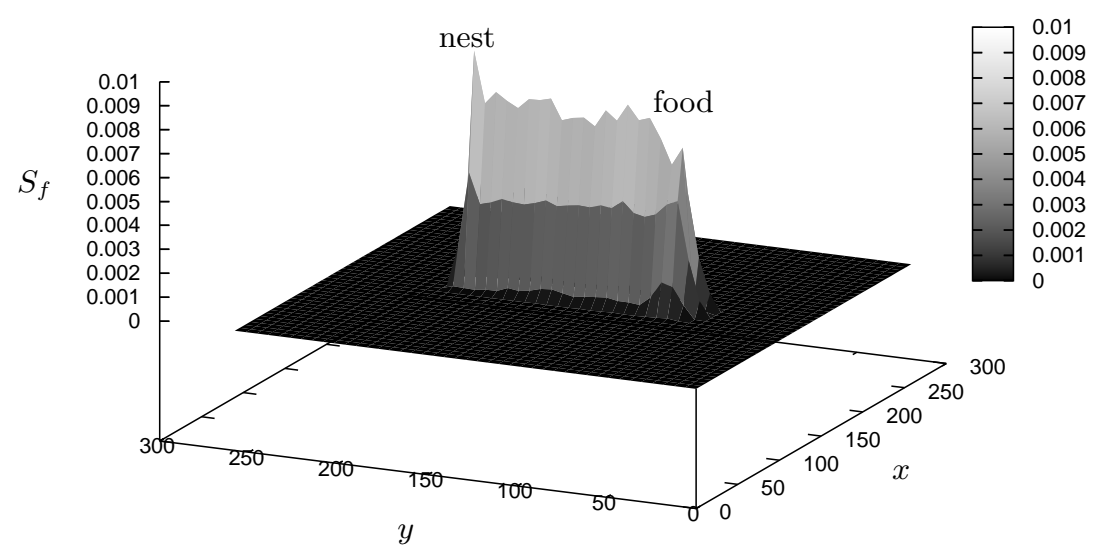

(b) Averaged over 30 simulation runs with $N=100$ agents.

Fig. 1. Distribution of $S_{f}$ for $D \approx 0.55$. 
Furthermore, we are interested in the resulting flow of food that is the same as the rate of robots that perform a transition from $s_{n}$ to $s_{f}$ per time. The amount of robots that perform a transition at the boundaries of the nest and the food per time in our model gives a good prediction of the flow. Another estimate of the converged flow, that turned out to be less sensitive to correctly measuring the diffusion, is obtained by integrating the densities of the steady-state over a line in the mid between nest and food, that is orthogonal to the shortest path between nest and food. Say the result of this integration is $I$ then the prediction of the flow for the given diffusion constant $D$ would be $I(1-D)=I \alpha$. This is a good approximation because the component of the pheromone gradient in the direction orthogonal to this plane is typically small. Thus the diffusion in this direction will also be small following Fick's First Law that gives the diffusion flux in the steady state: $J(\mathbf{r})=-D \frac{\partial P(\mathbf{r})}{\partial \mathbf{r}}$. This is the method we used to predict the flow.

In every simulation run, we drew 36 samples equidistant in time after a transient. The results are averaged over the samples of 30 runs. More runs would be desirable for a better statistical significance, however, due to limited resources and the high computational demand of the simulation, that could not be achieved. Two sets of simulation runs with two different swarm sizes $N \in\{100,150\}$ were performed and we could only reach diffusion rates in the interval $0.5<D<0.9$. The flow decreases with the diffusion, as expected (see Fig. 2, the error bars show the $95 \%$ confidence interval based on the $t$ distribution).

In order to maximize the flow and following these results one would like to set the diffusion as low as possible. However, our observations of the simulation indicate that the lower the diffusion rate is the more unstable the system becomes. Please note that the situation described here will only occur in simulations that implement interference effects between agents and an unbiased gradient ascent. If the robots follow the pheromone gradient $\frac{\partial P_{f}}{\partial r}$ very greedily, it becomes highly probable that they accumulate at certain spots. This might be caused by and lead itself to local maxima in the pheromone intensities $P_{f}$, where robots of state $s_{f}$ are attracted. These groups of robots block others in state $s_{n}$ traveling in the opposite direction. Hence, the local intensities $P_{f}$ are reinforced and more and more robots accumulate at this spot. As a consequence, the flow of food might even break down temporarily (compare to Fig. 3). This fact is not directly represented by the flow prediction of our model as it only gives the average flow of a functioning swarm without modeling effects of interference. While in the flow diagram of the model the intensity and number of oscillations only increases with decreasing diffusion, the batch-wise flow caused by robots moving in large groups and the temporary stop of the flow appears as an overshooting in the flow diagram of the simulation. These observations give rise to another measure introduced in the following.

As a qualitative measure for the instability of the system we use a damping constant $d$ as it is used in control theory. The run of the flow over time can 


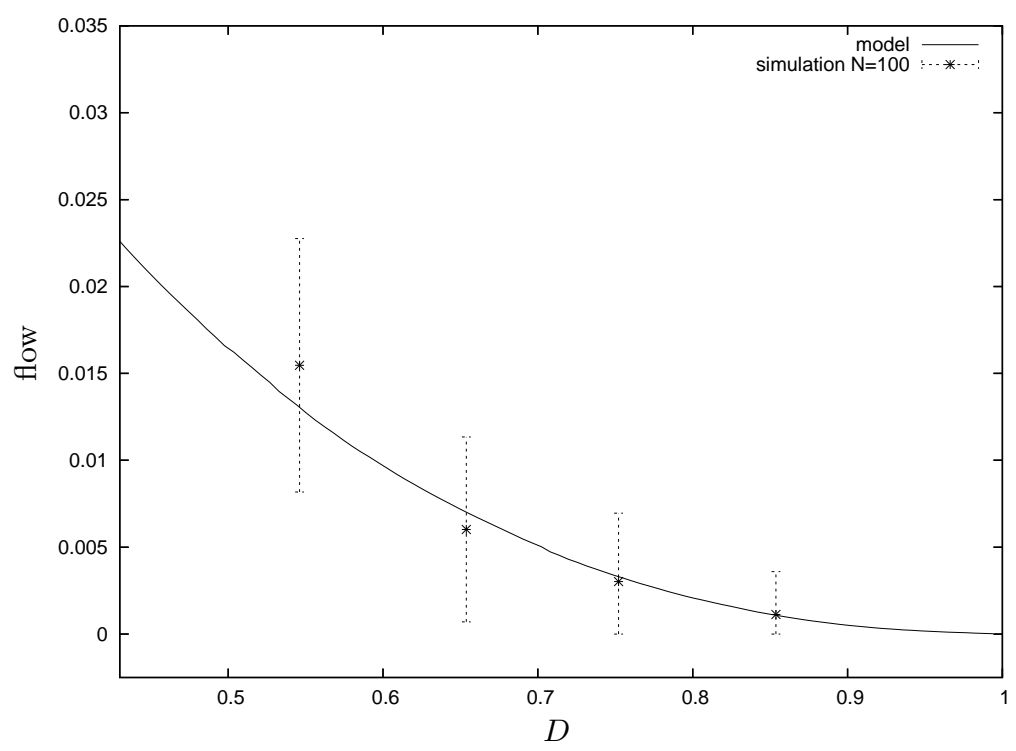

(a) 100 agents

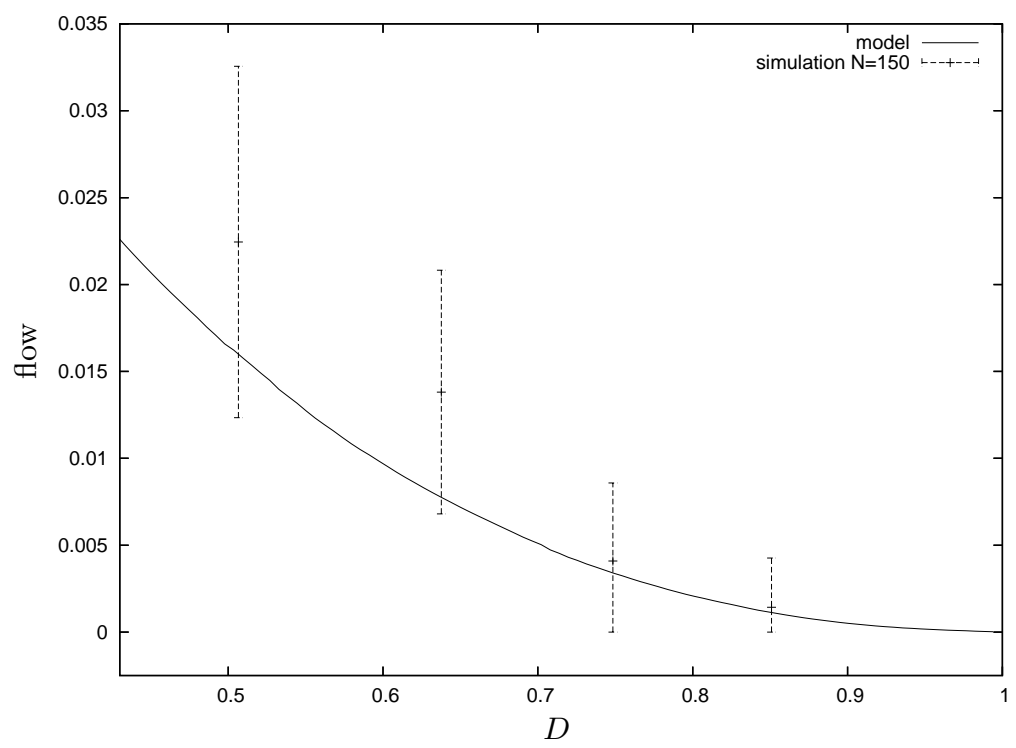

(b) 150 agents

Fig. 2. Average normalized flow of food as a function of the diffusion $D$. 


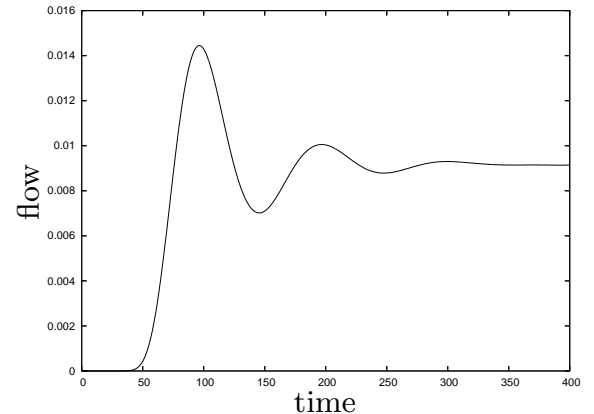

(a) model

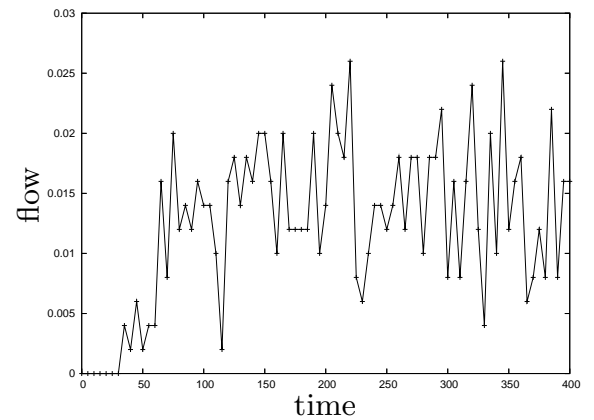

(c) single simulation run showing stable (d) single simulation run showing unstable behavior

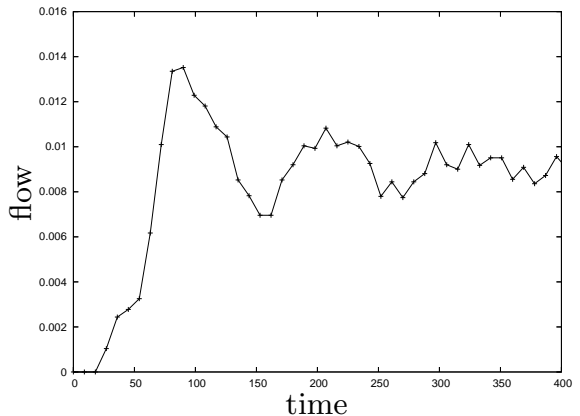

(b) averaged over 30 simulation runs

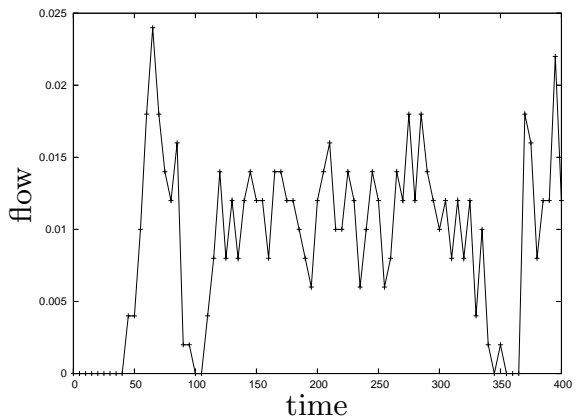
behavior

Fig. 3. Food flow over time. 
be interpreted as a step response and modeled by a 2nd-order lag element (PT2). Such an element is stable for $0<d<1$ (underdamped) as well as for $d \geq 1$ (overdamped) and shows oscillating behavior in the former region. Our observations showed that as well in the model as in the simulation the number and amplitude of these oscillations decrease with the diffusion.

To get reasonable results, we had to average over all available simulation results and thus cannot give any statistical measure. For too high numbers of robots leading to high densities the effects by interference induce a higher damping for low diffusion rates than predicted. However, a trend can be noticed in Fig. 4 and shows that the damping of the P-T2 element can serve as a qualitative model for the stability of the swarm behavior.

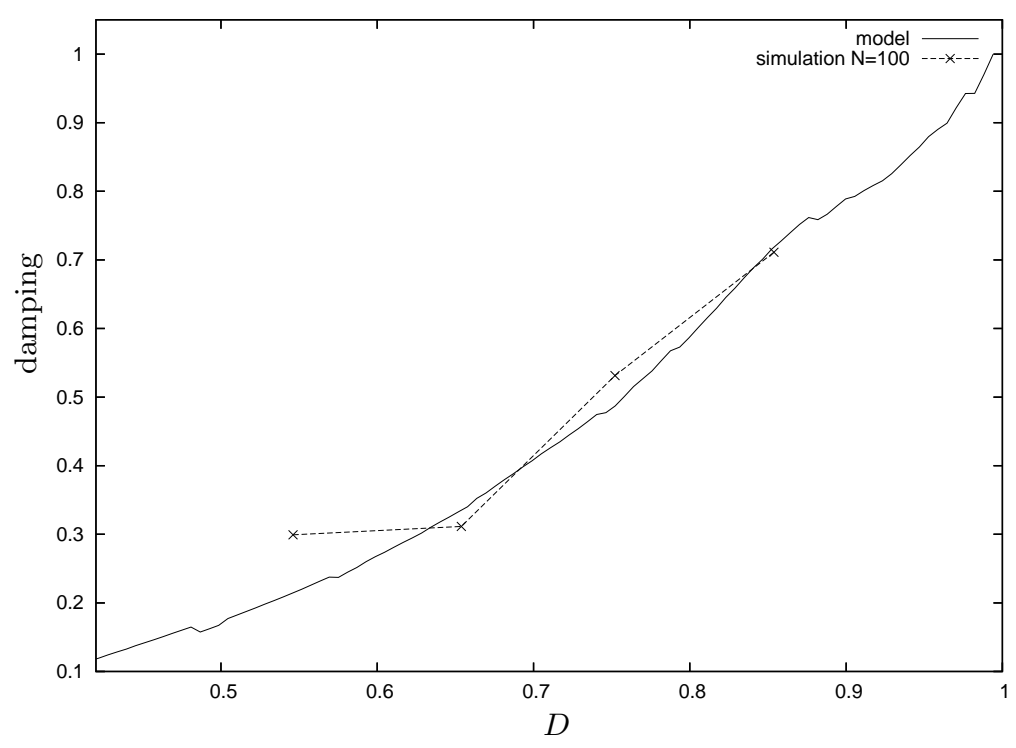

Fig. 4. Damping as a function of the diffusion $D$.

\section{Conclusion and Outlook}

The application of this analytical model to the foraging scenario has shown that the overall behavior of large groups of robots can be predicted well and described by the use of mathematical methods. Compared to the simulation the average behavior is computed faster by three or more orders of magnitude. The diffusion constant can be measured by the simulation within minutes. However, this constant models abstractly a variety of basic behaviors like exploration and collision avoidance. A direct connection to the control software does usually not 
exist. Thus a found optimal diffusion constant can only serve as a broad guideline for the development of the software.

There are a variety of possible extensions to this model: At first, it would be desirable to model also the interferences between robots that accumulate densely at one place, to investigate the characteristics of scalability in this scenario. This might be achieved by combining this model and the one presented in [10] or by better approximations of the diffusion process (Wilke or Maxwell-Stefan diffusion). Furthermore, a scenario with two dynamic pheromones and obstacles as well as the adaptability to food sources that change in position or quality over time could be investigated.

We plan to implement the presented scenario on real robots following the approach of Sugawara et al., that implemented it for a number of up to three robots [20]. At our institute, a swarm of 40 Jasmine robots is available (see [6]), that will be extended soon. We also have a combination of a video projector and a video camera installed above the arena. Additionally, light sensors on the top of the robots are under development and will be used by the robots to perceive the virtual pheromone gradient, i.e. a light gradient. The robots are able to drop pheromones by lighting an LED on their top, which will be detected by a computer connected to the camera and it will adapt the image projected onto the arena accordingly. With this setting it is easily possible to simulate scenarios physically that would actually need more technical overhead, e.g. following a gradient of temperature or gas concentrations. Even the combination of the two paradigms of self-organization and central control (on demand) might lead to synergies for example in microassembly. Self-organizing techniques provide robustness and scalability while central control techniques provide highest accuracy if needed.

Acknowledgments. The authors want to thank the anonymous referees for their excellent report, which has largely improved the clarity and content of the paper. This work was partially supported by the German Research Foundation (DFG) within the Research Training Group GRK 1194 Self-organizing SensorActuator Networks.

\section{References}

1. Arkin, R. C.: Motor schema based mobile robot navigation. International Journal of Robotics Research 8 (1989) 92-112

2. Bonabeau, E., Thraulaz, G., Fourcassi, V. and Deneubourg, J.: The phase-ordering kinetics of cemetery organization in ants. Technical Report 98-01008, Santa Fe Institute, 1998.

3. Cole, B.J. and Cheshire, D.: Mobile Cellular Automata Models of Ant Behavior: Movement Activity of Leptothorax allardycei. American Naturalist, Vol. 148 (1996), pp. $1-15$

4. Deneubourg, J.L., Aron, S., Goss, S. and Pasteels, J.M.: The self-organizing exploratory pattern of the Argentine ant. Journal of Insect Behavior 3 (1990) 159-168 
5. Goss, S., Aron, S. , Deneubourg, J.L. and Pasteels, J.M.: Self-organized shortcuts in the Argentine ant. Naturwissenschaften 76 (1989) 579-581

6. Jasmine Robot - Project Website. http://www.swarmrobot.org/. (2006)

7. Khatib, O.: Real-time obstacle avoidance for manipulators and mobile robots. International Journal of Robotics Research 5 (1986) 90-98

8. Klein, J.: Continuous 3D Agent-Based Simulations in the breve Simulation Environment. In: Proceedings of NAACSOS Conference (North American Association for Computational, Social, and Organizational Sciences). (2003)

9. Lerman, K.: A model of adaptation in collaborative multi-agent systems. Adaptive Behavior 12 (2004) 187-198

10. Lerman, K., Galstyan, A. Mathematical Model of Foraging in a Group of Robots: Effect of Interference. Autonomous Robots 13 (2002) 127-141

11. Lerman, K., Jones, C., Galstyan, A. and Mataric, M.: Analysis of Dynamic Task Allocation in Multi-Robot Systems. Int. J. of Robotics Research (2006)

12. Lerman, K., Martinoli, A. and Galstyan, A.: A Review of Probabilistic Macroscopic Models for Swarm Robotic Systems In: Sahin, E., Spears, W. (eds.): Swarm Robotics Workshop: State-of-the-art Survey. Springer-Verlag, Berlin Heidelberg New York (2005)

13. Martinoli, A., Easton, K. and Agassounon, W.: Modeling Swarm Robotic Systems: A Case Study in Collaborative Distributed Manipulation. In: Siciliano, B. (editor): Special Issue on Experimental Robotics, Int. Journal of Robotics Research 23 (2004) 415-436.

14. Panait, L. and Luke, S.: Ant Foraging Revisited. In: Pollack, J., Bedau, M., Husbands, P. and Ikegami, T. (eds.): ALife IX Proceedings. MIT Press, Cambridge (2004)

15. Schweitzer, F.: Brownian Agents and Active Particles. On the Emergence of Complex Behavior in the Natural and Social Sciences. Springer-Verlag, Berlin Heidelberg New York (2003)

16. Schweitzer, F.: Brownian Agent Models for Swarm and Chemotactic Interaction. In: Polani, D., Kim, J., Martinetz, T. (eds.): Fifth German Workshop on Artificial Life. Abstracting and Synthesizing the Principles of Living Systems. Akademische Verlagsgesellschaft Aka (2002)

17. Schweitzer, F., Lao, K., Family, F.: Active Random Walker Simulate Trunk Trail Formation by Ants. BioSystems 41 (1997) 153-166

18. Seyfried, J., Szymanski, M., Bender, N., Estana, R., Thiel, M. and Wörn, H.: The I-SWARM project: Intelligent Small World Autonomous Robots for Micromanipulation. In: Sahin, E. and Spears, W. (eds.): Swarm Robotics Workshop: State-of-the-art Survey. Springer-Verlag, Berlin Heidelberg New York (2005)

19. Spears, W.M. and Gordon, D.F.: Using Artificial Physics to control agents. In: IEEE International Conference on Information, Intelligence, and Systems. (1999)

20. Sugawara, K., Kazama, T. and Watanabe, T.: Foraging Behavior of Interacting Robots with Virtual Pheromone. In: Proceedings of 2004 IEEE/RSJ International Conference on Intelligent Robots and Systems. (2004)

21. Theraulaz, G., Gautrais, J., Camazine S. and Deneubourg, J.L.: The formation of spatial patterns in social insects: from simple behaviours to complex structures. Phil. Trans. R. Soc. Lond. A 361 (2003), 1263-1282 